\title{
Fractures of the neck of the fifth metacarpal bone. Medium-term results in 28 cases treated by percutaneous transverse pinning
}

\author{
V. Potenza*, R. Caterini, F. De Maio, S. Bisicchia, P. Farsetti \\ Department of Orthopaedic Surgery, University of Rome 'Tor Vergata', Rome, Italy
}

A R T I C L E I N F O

Article history:

Accepted 27 October 2011

\section{Keywords:}

Boxer's fracture

Transverse pinning

Metacarpal neck fracture

\begin{abstract}
A B S T R A C T
The purpose of this study was to report the medium-term results in 28 patients affected by closed displaced fractures of the neck of the fifth metacarpal bone (boxer's fracture) with an associated severe swelling of the hand, who were treated with percutaneous transverse K-wire pinning, to verify the effectiveness of this surgical treatment. We opted for this treatment in all cases in which malrotation of the fifth finger and volar angulation of the metacarpal head greater than $30^{\circ}$ were associated with a severe swelling of the hand. All the patients were reviewed clinically and radiologically at an average of 25 months after surgery. At the final follow-up, no patient reported residual pain. All patients had full extension of the fifth finger, except two in whom we observed a limitation of the extension of the fifth metacarpophalangeal (MP) joint of about $10^{\circ}$, without significant impairment of hand function. All patients had at least $90^{\circ}$ flexion of the fifth MP joint and full range of motion of the interphalangeal (IP) joints. No patient had rotational deformity of the fifth finger with a deficit of grip strength. At the final follow-up, a residual palmar angulation of the head of the fifth metacarpal was found in three patients, with a mean of $7^{\circ}$. The disabilities of the arm, shoulder and hand (DASH) scale had a mean value of 5 , and all patients considered their result as good or excellent. We recommend percutaneous transverse pinning in all boxer's fractures in which operative treatment is indicated, especially in patients with severe softtissue swelling. The surgical procedure is easy to perform, and surgical results are generally good.
\end{abstract}

(c) 2011 Elsevier Ltd. All rights reserved.
Fractures of the metacarpal bones are very common injuries of the skeletal system and, in approximately $50 \%$ of the cases, involve the neck of the fifth metacarpal bone. These fractures are frequently observed in active young men, occur in the dominant hand and are typical injuries of aggression (boxer's fractures). ${ }^{1-3}$ Surgical treatment is mandatory for severely displaced and unstable fractures to avoid impairment of hand function. In fact, malrotation or volar angulation of the metacarpal head may frequently cause reduction in the grip strength and lack of full extension of the fifth finger. ${ }^{3-7}$ The results of surgical treatment seem to be influenced by the preoperative condition of the soft tissues of the hand; therefore, in fractures associated with softtissue injuries, most authors suggest minimising the surgical trauma so as to obtain better results. ${ }^{3,6}$

Berkman and Miles ${ }^{8}$ first described transverse wire fixation to intact metacarpals for treatment of unstable fractures of the metacarpal bones. The purpose of this retrospective study was to report the medium-term results in 28 patients affected by

\footnotetext{
* Corresponding author. Tel.: +39 062090 3466; fax: +39 0620903465 . E-mail address: vitopotenza2@virgilio.it (V. Potenza).
}

displaced fracture of the neck of the fifth metacarpal (boxer's fracture), with an associated severe swelling of the surrounding soft tissues, who were treated by percutaneous transverse pinning. ${ }^{8}$

\section{Materials and methods}

In a pool of 88 consecutive fractures of the metacarpals surgically treated at our hospital between 2004 and 2008, 44 were displaced fractures of the neck of the fifth metacarpal bone (boxer's fracture). At diagnosis, 35 of these fractures showed a severe swelling of the surrounding soft tissues; they were treated by closed reduction and percutaneous transverse K-wire pinning. All 35 patients were invited to our hospital for a clinical and radiological follow-up, but only 28 were able to return for examination. There were 25 male and three female patients; the right hand was involved in 24 cases and the left in four. The average age of the patients was 38.4 years (range 15-71 years). The mechanism of trauma was a direct blow in 25 patients and a scooter accident in three. In all patients, a rotational deformity of the fifth finger and/or a palmar angulation of the fracture $\geq 30^{\circ}$ were present. Rotational displacement of the fifth ray was 
evaluated clinically: malrotation of the fifth metacarpal was diagnosed when the fifth finger was not oriented towards the scaphoid tubercle in flexion, and if the little finger was not parallel to the other fingers in extension. The palmar angulation of the metacarpal head was measured on the latero-oblique X-rays with a goniometer; it measured from $45^{\circ}$ to $90^{\circ}$, with an average of $63^{\circ}$. Soft-tissue swelling of the hand was considered severe when the knuckles of the fingers were not clinically detectable and the fovea sign was present on the dorsum of the hand.

The patients were operated on using a peripheral nerve block anaesthesia. Under fluoroscopy, we performed a closed reduction of the fracture by manipulation according to the Jahss manoeuvre ${ }^{9}$ and internal fixation using two K-wires $1.5 \mathrm{~mm}$ thick. The first Kwire was introduced percutaneously, proximally to the fracture and perpendicular to the ulnar border of the hand, and fixed from the fifth to the fourth metacarpal shaft, whilst the second K-wire was introduced distally to the fracture and parallel to the first wire, through the fifth and the fourth metacarpal heads. Since malrotation is usually difficult to assess under fluoroscopy, we verified correct rotation clinically. In flexion, the little finger should be oriented towards the scaphoid tubercle, whilst in extension, the fifth fingernail should be parallel to the other fingernails. Postoperatively, the hand was immobilised in a short-arm splint. Seven days after surgery, an X-ray of the hand was taken to detect any secondary displacement of the fracture. The K-wires and the splint were removed in the outpatient department after an average period of 5 weeks (range 4-6 weeks).

All the patients were reviewed clinically and radiographically from 13 to 37 months after surgery (average 25 months). From the clinical point of view, we evaluated the range of motion of the metacarpophalangeal (MP) and interphalangeal (IP) joints, the grip strength, and the possible presence of malrotation of the fifth finger and of the dropped-knuckle sign with lack of extension of the little finger. Moreover, we used disabilities of the arm, shoulder and hand (DASH) scale (0-100 points) to assess upper arm function. From the radiographic point of view, antero-posterior (AP) and latero-oblique X-rays were taken to assess the possible presence of a residual deformity of the fifth finger.

\section{Results}

In five cases, a minor local infection occurred at the site of introduction of the K-wires, and it was treated successfully with antibiotic therapy. No patient suffered secondary displacement of the fracture at the X-ray taken 7 days after surgery.

At the final follow-up, all patients were satisfied with the final result. No patient reported residual pain, and no patient showed the dropped-knuckle sign. All patients but two had full extension of the fifth finger. In those two patients, we observed a slight limitation of the extension of the fifth MP joint of less than $10^{\circ}$, although they did not report any significant impairment of their hand function. All patients had at least $90^{\circ}$ flexion of the fifth MP joint and full range of motion of the IP joints. No patient had any clinically detectable rotational deformity of the fifth finger, with a deficit of grip strength. The DASH scale had a mean value of five points, ranging from one to eight points.

At the final X-ray examination, we never observed nonunion, avascular necrosis of the fifth metacarpal head or degenerative signs of the fifth MP joint. Preoperatively, the average palmar angulation of the head of the fifth metacarpal was $63^{\circ}$ (range 45$90^{\circ}$ ), whereas at the final follow-up a residual palmar angulation of the head of the fifth metacarpal was found in only three patients, averaging $7^{\circ}$ (range $5-13^{\circ}$ ) (Fig. 1 ). The difference between the value of the palmar angulation of the fifth metacarpal head, measured radiographically before and after treatment, was significant.

\section{Discussion}

The purpose of this retrospective study was to assess the medium-term clinical and radiographic results of percutaneous transverse fixation with $\mathrm{K}$-wires in displaced and unstable fractures of the neck of the fifth metacarpal bone (boxer's fractures). Boxer's fractures are very common injuries, ${ }^{2,9}$ which can cause impairment of hand function., ${ }^{5,10,11}$ When they heal in malrotation and/or in volar angulation of the metacarpal head, the result may be a loss of the grip strength and an extension deficit of the little finger. The management of boxer's fracture is still controversial; however, surgical treatment is always indicated in severely displaced and unstable fractures. Various operative techniques have been proposed for the surgical treatment of these fractures, ${ }^{1,12-15}$ although transverse pinning, first described by Berkman and Miles, ${ }^{8}$ is currently one of the surgical procedures of choice. We report the results obtained in 28 patients with a displaced fracture of the neck of the fifth metacarpal, treated surgically using percutaneous transverse fixation with two K-wires to the intact fourth metacarpal and followed-up at least 13 months after surgery. Following the criteria of Ali et al. ${ }^{10}$ and of Birndorf et al., ${ }^{5}$ we only operated on patients with rotational displacement of the fifth finger and/or palmar angulation of the fracture of $30^{\circ}$ or more. At follow-up, no patient had any clinically detectable rotational deformity of the fifth finger with a consequent deficit of the grip strength, and at the final radiographic examination we never observed nonunion, avascular necrosis of the fifth metacarpal head or abnormalities of the MP joint. In spite of a mean period of immobilisation of 5 weeks, only two patients showed a minimal deficit of the extension of the little finger. At the radiographic examination, a mild residual palmar angulation of the fifth metacarpal head was observed in three patients. None of these last five patients reported significant impairment of their hand function. The residual palmar angulation, observed in three cases, could be related to the fact that one single K-wire, inserted through the distal fragment, might not control rotation of the fragment. To avoid this residual deformity of the fifth finger, a third K-wire (in addition to one distal and one proximal) could be inserted in the distal fragment to better stabilise the reduction obtained. All our patients, at surgery, presented a severe swelling of the surrounding soft tissues of the hand. We believe that the presence of a severe swelling of the soft tissues of the hand is one more reason to choose this type of treatment which minimises the surgical trauma.

To the best of our knowledge, several papers have been published on the transverse K-wire fixation technique for treatment of fractures of the metacarpals, ${ }^{6,7,16,17}$ but no mediumor long-term results have been reported. In their short-term retrospective study with only 3 months of follow-up, Galanakis et al. $^{6}$ reported excellent functional and radiographic outcomes in a series of patients affected by unstable metacarpal fractures treated with percutaneous transverse fixation with K-wires. Winter et al., ${ }^{7}$ in their short-term retrospective study, with a mean follow-up of 2.7 months, reported that in the boxer's fracture intramedullary pinning gave better functional outcomes than transverse pinning, although they concluded that intramedullary pinning is technically more demanding than transverse pinning and the surgeon has a more definite learning curve.

Intramedullary pinning and internal fixation with locking plates are the other two common methods of treatment for boxer's fracture. Recently, in a comparative study on fifth metacarpal neck fracture fixation, Facca et al. ${ }^{15}$ reported better results in the series stabilised with intramedullary K-wire in comparison to a second series treated with locking plates and immediate mobilisation. In this second series treated with locking plates, the authors reported a deficit, despite immediate mobilisation, of the MP joint due to the adherences of the extensor 

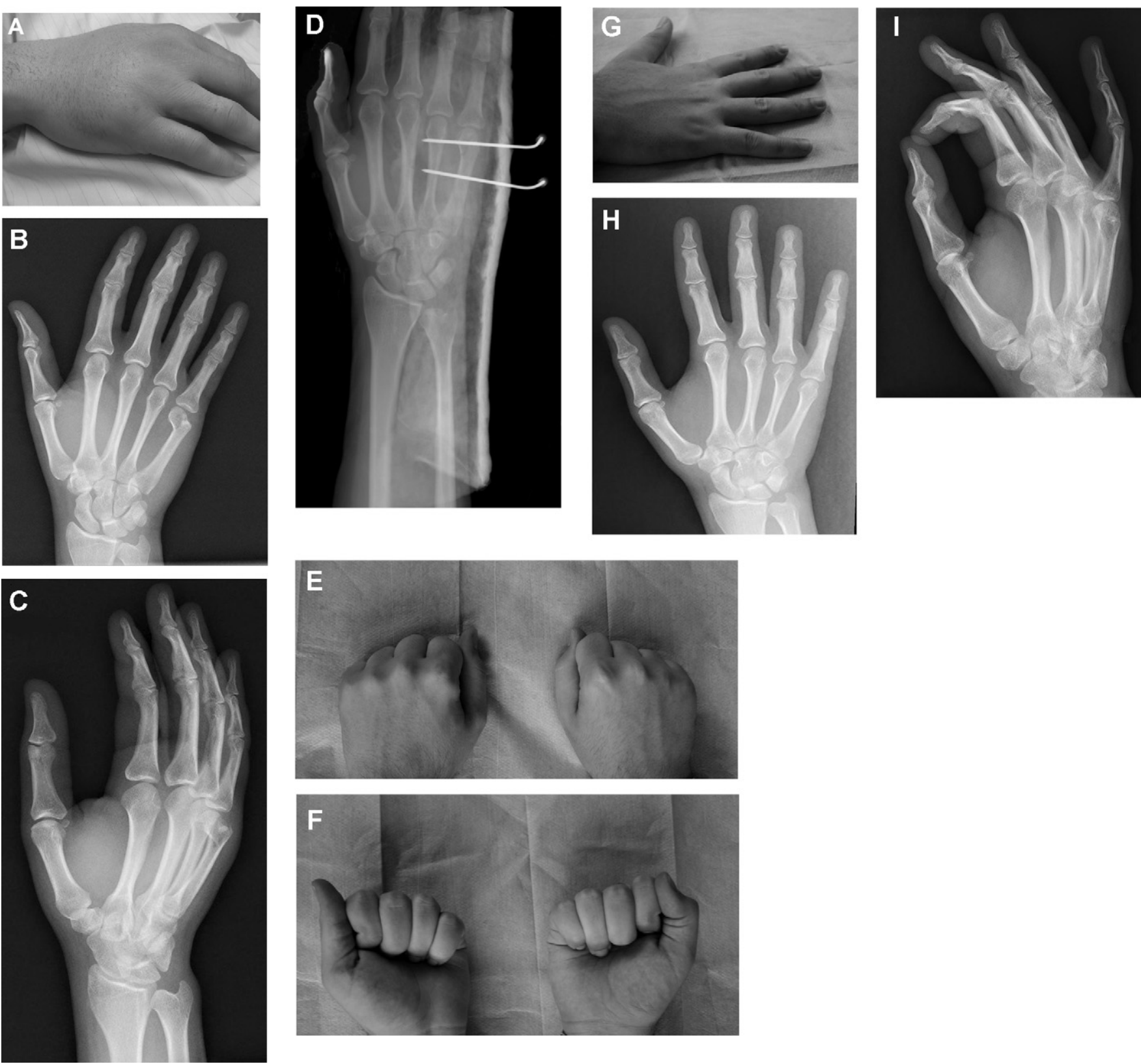

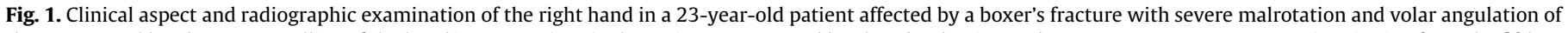

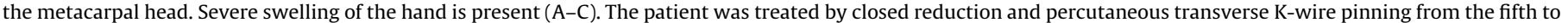

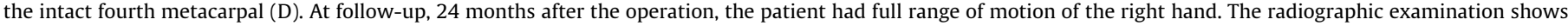
perfect healing of the fracture without malrotation or volar angulation of the metacarpal head (E-I).

apparatus. In addition, they reported a case of avascular necrosis of the metacarpal head in the same series. In a short-term review, with a mean follow-up of 9 months, Calder et al. ${ }^{12}$ reported excellent results in six patients treated for displaced boxer's fractures, stabilised by a modified anterograde intramedullary pin. We disagree with this treatment because we believe that by using only one K-wire, although modified, the risk of a secondary rotational displacement of the fracture is extremely high. Foucher et al. ${ }^{13,14}$ reported excellent functional results in 62 patients affected by displaced fifth metacarpal neck fracture, surgically treated by closed reduction and anterograde internal fixation with three pre-bent K-wires (Bouquet osteosynthesis). As reported by the authors themselves, this is a very difficult surgical technique, which requires a long learning curve. Neither Calder et al. ${ }^{12}$ nor Foucher et al. ${ }^{13,14}$ mentioned the preoperative condition of the surrounding soft tissue of the hand, which greatly influences the outcome after surgery, as reported by other authors. ${ }^{8}$ Some authors ${ }^{15,18}$ reported good results using a retrograde intramedullary pinning for the treatment of boxer's fracture. In agreement with other authors, we believe that retrograde fixation of the fifth metacarpal bone may cause MP joint stiffness, with a frequent limitation of the extension of the fifth finger, due to the trauma of the extensor hood and the collateral ligaments during the wire insertion. $^{12}$

The limitations of our study are that it is a retrospective study which included a relatively small number of patients, without a control series of patients treated with a different method. However, on the basis of our final results, we believe that transverse pinning fixation to the intact fourth metacarpal bone for displaced boxer's fractures is a valid and easy surgical technique, 
with minimised surgical trauma. In our opinion, anterograde intramedullary nailing using more than one pin remains the other valid option of treatment, although this surgical technique is more difficult. Internal fixation with plates or retrograde intramedullary nailing should, if possible, be avoided.

In conclusion, we recommend percutaneous transverse pinning in all boxer's fractures in which surgery is indicated, especially when severe swelling of the hand is present.

\section{Conflict of interest}

The authors report no conflict of interest.

\section{References}

1. Green DP, Rowland SA. Fractures and dislocations in the hand. In: Rockwood Jr CA, Green DP, Bucholz RW, editors. Fractures in adults. 3rd ed. Philadelphia: JB Lippincott; 1991. p. 441-562.

2. Greer SE, Williams JM. Boxer's fracture: an indicator of intentional and recurrent injury. Am J Emerg Med 1999;17:357-60.

3. Kozin SH, Thoder JJ, Lieberman G. Operative treatment of metacarpal and phalangeal shaft fractures. J Am Acad Orthop Surg 2000;8:111-21.

4. McKerrell J, Bowen V, Johnston G, Zondervan J. Boxer's fractures - conservative or operative management? J Trauma 1987;27:486-90.

5. Birndorf MS, Daley R, Greenwald DP. Metacarpal fracture angulation decreases flex or mechanical efficiency in human hands. Plast Reconstr Surg 1997;99:1079-83.
6. Galanakis I, Aligizakis A, Katonis P, Papadokostakis G, Stergiopoulos K, Hadjipavlou A. Treatment of closed unstable metacarpal fractures using percutaneous transverse fixation with Kirschner wires. J Trauma 2003;55:509-13.

7. Winter M, Balaguer T, Bessière C, Carles M, Lebreton E. Surgical treatment of the boxer's fracture: transverse pinning versus intramedullary pinning. J Hand Surg Eur Vol 2007;32:709-13.

8. Berkman EF, Miles GH. Internal fixation of metacarpal fractures exclusive of the thumb. J Bone Joint Surg Am 1943;25:816-21.

9. Jahss SA. Fractures of the metacarpals. A new method of reduction and immobilization. J Bone Joint Surg Am 1938;20:178-86.

10. Ali A, Hamman J, Mass DP. The biomechanical effects of angulated boxer's fractures. J Hand Surg Am 1999;24:835-44.

11. Meunier MJ, Hentzen E, Ryan M. Predicted effects of metacarpal shortening on interosseous muscle function. J Hand Surg Am 2004;29:689-93.

12. Calder JDF, O'Leary S, Evans SC. Antegrade intramedullary fixation of displaced fifth metacarpal fractures. Injury 2000;31:47-50.

13. Foucher $G$, Chemorin C, Sibilly A. Nouveau procédé d'ostéosynthèse original dans les fractures du tiers distal du cinquième métacarpien. Nouv Presse Med 1976;5:1139-40.

14. Foucher G. "Bouquet" osteosynthesis in metacarpal neck fractures. A series of 66 patients. J Hand Surg Am 1995;20:S86-90.

15. Facca S, Ramdhian R, Pelissier A, Diaconu M, Liverneaux P. Fifth metacarpal neck fracture fixation: locking plate versus K-wire? Orthop Traumatol Surg Res 2010;96:506-12.

16. Lamb DW, Abernethy PA, Raine PA. Unstable fractures of the metacarpals. A method of treatment by transverse wire fixation to intact metacarpals. Hand 1973;5:43-8.

17. Mitz V, Richard JC, Ohanna J, Vilain R. Intéret de l'ostéosynthèse par brochage transversal externe des fractures du cinquième métacarpien. Rev Chir Orthop 1981;67:571-6.

18. Lord RE. Intramedullary fixation of metacarpal fractures. $J$ Am Med Assoc 1957;164:1746-9. 